Article

\title{
Effects of Nonlinear Chirp on the Self-Phase Modulation of Ultrashort Optical Pulses
}

\author{
Akira Suda * and Takanori Takeda \\ Department of Physics, Faculty of Science and Technology, Tokyo University of Science, \\ 2641 Yamazaki, Noda, Chiba 278-8510, Japan \\ * Author to whom correspondence should be addressed; E-Mail: asuda@ph.noda.tus.ac.jp; \\ Tel.: +81-4-7122-1447; Fax: +81-4-7122-1495.
}

Received: 30 April 2012; in revised form: 28 May 2012 / Accepted: 29 May 2012 /

Published: 4 June 2012

\begin{abstract}
In this article, we analytically investigate the spectral broadening by self-phase modulation of strongly chirped optical pulses. The dispersion due to the nonlinear optical process is expressed as functions of a linear and a nonlinear initial chirp. As a result, it is found that the third-order dispersion strongly depends on the initial linear chirp and the nonlinearity for self-phase modulation.
\end{abstract}

Keywords: ultrashort optical pulse; self-phase modulation; nonlinear chirp

\section{Introduction}

Optical pulse compression using self-phase modulation (SPM) and subsequent dispersion compensation is a widely used technique for ultrashort pulse generation, first demonstrated with an optical fiber in 1987 [1]. However, the energy generated in that case was several nJ. Then, the advent of gas-filled hollow fibers enabled us to generate few-cycle optical pulses with energies in excess of $100 \mu \mathrm{J}$ [2]. This development has led to the generation of attosecond x-ray pulses by high-order harmonic conversion of few-cycle optical pulses [3]. One of the main features of the hollow fiber compression technique is that a broadband spectrum together with a monotonic spectral phase suitable for dispersion compensation can readily be obtained by SPM. For this reason, monocycle to few-cycle pulses can be generated without any serious technical difficulties. Other features are the excellent beam quality and the focusability, which arise because of SPM taking place inside the hollow fiber during propagation. On the other hand, the drawback is energy scaling. As the energy incident on a 
hollow fiber increases, the coupling to the fiber mode is degraded by the poor beam quality due to self-focusing and multi-photon ionization, both of which take place near the entrance to the fiber. In order to avoid this problem, we have developed a pressure-gradient hollow fiber technique that can generate TW-class few-cycle pulses [4]. Further increases in energy require suppression of multi-photon ionization during propagation inside the fiber. The use of chirped pulses in spectral broadening by SPM was proposed as a solution to this problem and some experimental results indicated that this was possible as long as the pulse intensity was suppressed below the ionization threshold [5-7]. In order to realize this, the key technology is dispersion compensation over a wide range of the spectrum corresponding to a few optical cycles in the time domain. For this opportunity, we need to understand the relationship between the initial chirp imposed on the incident pulse and the dispersion after spectral broadening by SPM.

In this article, we present analytical expressions derived for spectral broadening by chirped pulse SPM and investigate the chirp of the output pulse. In particular, we argue that the influence of nonlinear chirp on the third-order dispersion, which must be compensated for in order to generate ultrashort pulses in the few optical cycle regime.

\section{Analytical Derivation}

\subsection{Self-Phase Modulation by a Linearly Chirped Pulse}

We consider that an optical pulse is subject to temporal phase modulation depending on its intensity profile, i.e., self-phase modulation (SPM). Assuming that the temporal profile is Gaussian, the Fourier-transform-limited (FTL) pulse is represented in the time and frequency domains, respectively, as,

$$
\begin{gathered}
E_{1}(t)=E_{0} \exp \left(-a t^{2}\right) \\
\tilde{E}_{1}(\omega)=\frac{E_{0}}{\sqrt{2 a}} \exp \left(-\frac{\omega^{2}}{4 a}\right)
\end{gathered}
$$

where $a=2 \ln 2 / \Delta t^{2}$ and $\Delta t$ is the pulsewidth (full width at half maximum: FWHM). Applying a group delay dispersion (GDD) $\phi^{\prime \prime}$ to Equations (1) and (2), the corresponding chirped pulses are expressed in the frequency and time domains, respectively, as follows.

$$
\begin{gathered}
\tilde{E}_{2}(\omega)=\frac{E_{0}}{\sqrt{2 a}} \exp \left[-\frac{\omega^{2}}{4 a}\left(1+i \Phi^{\prime \prime}\right)\right] \\
E_{2}(t)=\frac{E_{0}}{\sqrt{1+i \Phi^{\prime \prime}}} \exp \left(-\frac{a t^{2}}{1+i \Phi^{\prime \prime}}\right)
\end{gathered}
$$

Here, $\Phi^{\prime \prime}$ is the normalized GDD given by

$$
\Phi^{\prime \prime}=2 a \phi^{\prime \prime}
$$

which is a dimensionless quantity roughly equal to a pulse stretching ratio with regard to the FTL pulse having the same spectral width. For example, $\Phi^{\prime \prime}=10$ implies that the pulse is stretched by a factor of approximately 10 . 
When a linearly chirped pulse is subject to SPM, the temporal profile of the optical intensity is given by

$$
I_{2}(t)=I_{0} \exp \left(-\frac{2 a t^{2}}{1+\Phi^{\prime 2}}\right) \approx I_{0}\left(1-\frac{2 a t^{2}}{1+\Phi^{\prime 2}}\right)
$$

where $I_{0}$ is the peak intensity. It is noted that in Equation (6) the main part of the pulse can be approximated by a quadratic function. The nonlinear refractive index is expressed as follows.

$$
n(t) \approx n_{0}+n_{2} I_{0}-n_{2} I_{0} \frac{2 a t^{2}}{1+\Phi^{\prime 2}}
$$

Considering only the time-dependent term, i.e., the third term, Equation (4) can be rewritten as,

$$
E_{3}(t)=\frac{E_{0}}{\sqrt{1+i \Phi^{\prime \prime}}} \exp \left[-\frac{a t^{2}}{1+\Phi^{\prime 2}}\left\{1-i\left(2 B+\Phi^{\prime \prime}\right)\right\}\right]
$$

where $B\left(=\frac{2 \pi}{\lambda_{0}} n_{2} I_{0} L\right)$ is the $B$-integral, $\lambda_{0}$ is the center wavelength, and $L$ is the interaction length. The Fourier transform of Equation (8) yields

$$
\tilde{E}_{3}(\omega)=\frac{E_{0}}{\sqrt{2 a}} \sqrt{\frac{1-i \Phi^{\prime \prime}}{1-i\left(2 B+\Phi^{\prime \prime}\right)}} \exp \left[-\frac{\omega^{2}\left(1+\Phi^{\prime 2}\right)}{4 a\left\{1-i\left(2 B+\Phi^{\prime \prime}\right)\right\}}\right]
$$

The GDD of the output pulse resulting from SPM can be obtained from the imaginary part of the exponential function as

$$
\Phi_{\text {OUT }}^{\prime \prime}=\frac{\left(1+\Phi^{\prime 2}\right)\left(2 B+\Phi^{\prime \prime}\right)}{1+\left(2 B+\Phi^{\prime \prime}\right)^{2}} \Phi^{\prime \prime}
$$

where as the real part gives the spectral width (FWHM)

$$
\Delta \omega=\frac{4 \ln 2}{\Delta t} \sqrt{\frac{1+\left(2 B+\Phi^{\prime \prime}\right)^{2}}{1+\Phi^{\prime 2}}}
$$

It is noted that $\Phi_{O U T}^{\prime \prime}$ is a dimensionless normalized quantity, being the same as in Equation (5).

\subsection{Self-Phase Modulation by a Nonlinearly Chirped Pulse}

Applying both GDD and third-order dispersion (TOD) $\phi^{\prime \prime \prime}$ to a FTL pulse, the chirped pulse is expressed in the frequency and time domains, respectively, as follows.

$$
\begin{gathered}
\tilde{E}_{4}(\omega)=\frac{E_{0}}{\sqrt{2 a}} \exp \left[-\frac{\omega^{2}}{4 a}\left(1+i \Phi^{\prime \prime}\right)-i \frac{\omega^{3} \Phi^{\prime \prime \prime}}{12 a \omega_{0}}\right] \\
E_{4}(t)=\frac{E_{0}}{\sqrt{1+i \Phi^{\prime \prime}}} \exp \left[-\frac{a t^{2}}{1+i \Phi^{\prime \prime}}-\frac{2 \Phi^{\prime \prime \prime}\left\{1-3 \Phi^{\prime \prime 2}-i \Phi^{\prime \prime}\left(3-\Phi^{\prime 2}\right)\right\}}{3 \omega_{0}\left(1+\Phi^{\prime 2}\right)^{3}} a^{2} t^{3}\right]
\end{gathered}
$$


Here, $\Phi^{\prime \prime}$ is the normalized TOD given by

$$
\Phi^{\prime \prime \prime}=2 a \omega_{0} \phi^{\prime \prime \prime}
$$

where $\omega_{0}$ is the center wavelength. It should be noted that this is also a dimensionless quantity. Although the Fourier transform of the exponential function including the cubic function is the Airy function, we use following approximations assuming that the contribution of the cubic term is relatively small, i.e., $\left|b t^{2}\right|>\left|c t^{3}\right|$ and $\left|b \omega^{2}\right|>\left|c \omega^{3}\right|$ in the following equations, respectively.

$$
\begin{gathered}
\mathcal{F}\left[\exp \left(-b t^{2}-c t^{3}\right)\right] \approx \frac{1}{\sqrt{2 b}} \exp \left[-\frac{\omega^{2}}{4 b}-i c\left(\frac{\omega}{2 b}\right)^{3}\right] \\
\mathcal{F}^{-1}\left[\exp \left(-b \omega^{2}-i c \omega^{3}\right)\right] \approx \frac{1}{\sqrt{2 b}} \exp \left[-\frac{t^{2}}{4 b}-c\left(\frac{t}{2 b}\right)^{3}\right]
\end{gathered}
$$

The same treatment given in the previous section is applied to Equation (13), including the time-dependent refractive index.

$$
\begin{aligned}
E_{5}(t)=\frac{E_{0}}{\sqrt{1+i \Phi^{\prime \prime}}} \exp [ & -\frac{a t^{2}}{1+\Phi^{\prime \prime 2}}\left\{1-i\left(2 B+\Phi^{\prime \prime}\right)\right\} \\
& \left.-\frac{2 \Phi^{\prime \prime \prime}\left\{1-3 \Phi^{\prime \prime 2}-i\left(2 B\left(1-3 \Phi^{\prime \prime 2}\right)+\Phi^{\prime \prime}\left(3-\Phi^{\prime \prime 2}\right)\right)\right\}}{3 \omega_{0}\left(1+\Phi^{\prime 2}\right)^{3}} a^{2} t^{3}\right]
\end{aligned}
$$

The Fourier transform of Equation (17) based on Equation (16) yields

$$
\begin{gathered}
\tilde{E}_{5}(\omega)=\frac{E_{0}}{\sqrt{2 a}} \sqrt{\frac{1-i \Phi^{\prime \prime}}{1-i\left(2 B+\Phi^{\prime \prime}\right)}} \exp \left[-\frac{\omega^{2}}{4 a\left\{1-i\left(2 B+\Phi^{\prime \prime}\right)\right\}}\left(1+\Phi^{\prime 2}\right)\right. \\
\left.-i \frac{\Phi^{\prime \prime \prime}\left\{1-3 \Phi^{\prime \prime 2}-i\left(2 B\left(1-3 \Phi^{\prime \prime 2}\right)+\Phi^{\prime \prime}\left(3-\Phi^{\prime \prime 2}\right)\right)\right\}}{12 a \omega_{0}\left\{1-i\left(2 B+\Phi^{\prime \prime}\right)\right\}^{3}} \omega^{3}\right]
\end{gathered}
$$

The TOD of the output pulse resulting from SPM can be obtained from the imaginary part of the exponential function as

$$
\Phi_{O U T}^{\prime \prime \prime}=\frac{\left(1-3 \Phi^{\prime \prime 2}\right)\left\{1-\left(2 B+\Phi^{\prime \prime}\right)^{4}\right\}+2 \Phi^{\prime \prime}\left(1+\Phi^{\prime \prime 2}\right)\left(2 B+\Phi^{\prime \prime}\right)\left\{3-\left(2 B+\Phi^{\prime \prime}\right)^{2}\right\}}{\left\{1+\left(2 B+\Phi^{\prime \prime}\right)^{2}\right\}^{3}} \Phi^{\prime \prime \prime}
$$

It should be noted again that this is a dimensionless quantity. Both the GDD and spectral width of the output pulse are independent of the initial TOD, being the same as shown in Equations (10) and (11), respectively. This is because the contribution of TOD is assumed to be small. Strictly, the GDD and spectral width would be weak functions of the initial TOD. 


\subsection{Effects of Self-Steepening}

SPM is the lowest-order nonlinear phenomenon based on the optical Kerr effect that provides symmetric spectral broadening. The next higher-order nonlinear phenomenon is self-steepening, which gives asymmetric spectral broadening. Here, we consider self-steepening together with TOD, while GDD is neglected for simplicity.

A nonlinearly chirped pulse including TOD is expressed in the time domain as,

$$
E_{6}(t)=E_{0} \exp \left(-a t^{2}-\frac{2 \Phi^{\prime \prime \prime}}{3 \omega_{0}} a^{2} t^{3}\right)
$$

The optical intensity corresponding to the electric field is

$$
\left|E_{6}(t)\right|^{2}=E_{0}^{2} \exp \left(-2 a t^{2}-\frac{4 \Phi^{\prime \prime \prime}}{3 \omega_{0}} a^{2} t^{3}\right)
$$

From Equations (20) and (21)

$$
\frac{\partial}{\partial t}\left(\left|E_{6}(t)\right|^{2} E_{6}(t)\right)=-6 a t\left(1+\frac{\Phi^{\prime \prime}}{\omega_{0}} a t^{2}\right)\left|E_{6}(t)\right|^{2} E_{6}(t)
$$

and the temporal profile including the self-steepening can be represented by

$$
\begin{gathered}
E_{7}(t)=E_{0} \exp \left[-a(1-2 i B) t^{2}-\left\{\frac{\Phi^{\prime \prime \prime}}{3}(1-2 i B)+6 B\right\} \frac{2 a^{2} t^{3}}{\omega_{0}}\right. \\
\left.-\frac{8 B \Phi^{\prime \prime \prime}}{\omega_{0}^{2}}\left(1+\frac{a \Phi^{\prime \prime \prime} t}{\omega_{0}}\right) a^{3} t^{4}\right]
\end{gathered}
$$

Neglecting $t^{4}$ and higher-order terms, the Fourier transform of Equation (23) results in

$$
\begin{aligned}
\tilde{E}_{7}(\omega)=\frac{E_{0}}{\sqrt{2 a(1-2 i B)}} & \exp \left[-\frac{\omega^{2}}{4 a(1-2 i B)}+\frac{2\left(1-4 B^{2}\right) \Phi^{\prime \prime \prime}+9 B\left(3-4 B^{2}\right)}{3 a \omega_{0}\left(1+4 B^{2}\right)^{3}} B \omega^{3}\right. \\
& \left.-i \frac{\left(1-16 B^{4}\right) \Phi^{\prime \prime \prime}+18 B\left(1-12 B^{2}\right)}{6 a \omega_{0}\left(1+4 B^{2}\right)^{3}} \omega^{3}\right]
\end{aligned}
$$

The TOD of the output pulse resulting from SPM and self-steeping can be obtained from the imaginary part of the exponential function as

$$
\Phi_{O U T}^{\prime \prime \prime}=\frac{1-4 B^{2}}{\left(1+4 B^{2}\right)^{2}} \Phi^{\prime \prime \prime}+\frac{18 B\left(1-12 B^{3}\right)}{\left(1+4 B^{2}\right)^{3}}
$$

\section{Results and Discussion}

\subsection{Dispersion of a Linearly Chirped Pulse in SPM}

SPM of a FTL pulse results in an up-chirped pulse. This is also obvious from Equation (8). In this case, the sign of the GDD is positive, which requires a negative GDD for dispersion compensation. Here, we consider the SPM of strongly chirped pulses. 
Figure 1 shows the GDD of the output pulse as a function of initial GDD for various values of $B$. The GDD is almost proportional to the initial one, implying that the change in phase during SPM is small. It is also seen that the variation of the GDD with the $B$-integral is small. Based on these results, one can apply the dispersion management used in linear optical systems, such as chirped-pulse amplification, to spectral broadening by SPM. The only exception is the case in which a small negative GDD is imposed on the initial pulse, as seen by the peculiar points in Figure 1. In this case, the spectrum does not broaden but becomes a bit narrower, where the negative GDD in the down-chirped pulse cancels the positive GDD generated during the SPM process. There is a duality relationship between this situation and conventional pulse compression using spectral broadening and subsequent dispersion compensation, which can be understood from Equations (8) and (9). In actual fact, observation showed that the down-chirped pulse did not broaden the spectrum very much [6,7]. Therefore, if the down-chirped pulse is used to broaden the spectrum, the GDD applied to the initial pulse must be large enough to avoid this effect. On the other hand, the use of an up-chirped pulse does not have this difficulty and one can apply any GDD for chirped-pulse SPM, from a FTL pulse to a strongly chirped pulse.

Figure 1. Group Delay Dispersion (GDD) of the output pulse $\Phi_{O U T}^{\prime \prime}$ as a function of initial GDD $\Phi^{\prime \prime}$ for various values of $B$.

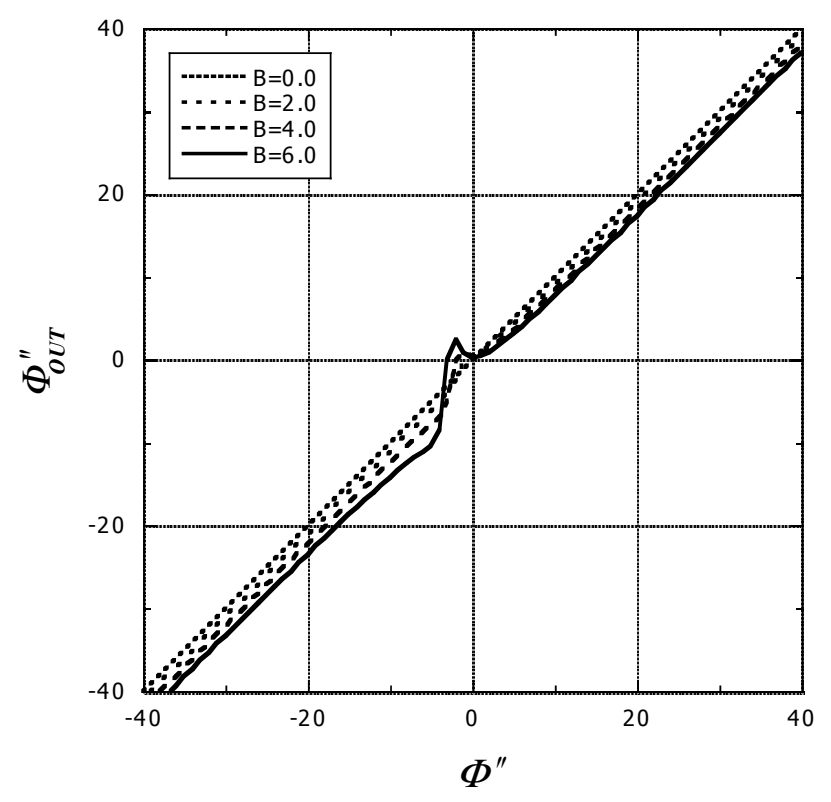

\subsection{Dispersion of a Nonlinearly Chirped Pulse in SPM}

In order to generate few-cycle pulses after spectral broadening by SPM, it is important to carefully compensate the phase of the output pulse, not only GDD but also TOD. The origin of TOD generated during the SPM process is asymmetry of the temporal intensity profile that gives a time-dependent nonlinear refractive index. One of the factors that provide an asymmetric temporal profile is a nonlinear chirp, i.e., TOD applied to the initial FTL pulse. Thus, the effects of TOD on the pulse shape appear before and after SPM, being related to the GDD. 
Figure 2 is the TOD of the output pulse based on Equation (19) as a function of initial TOD for various values of $B$. The initial GDD $\Phi^{\prime \prime}$ is (a) 10 , (b) -10 , and (c) 0 . In the case that a large amount of GDD is applied to the initial pulse, there is no great distinction between the TOD of the output pulse and the initial TOD, as shown in Figure 2(a,b). The slope tends to lessen slightly with increasing $B$. This tendency appears prominently in Figure 2(c). This is because SPM reduces the asymmetry in the temporal profile shown in the nonlinearly chirped pulse. As described above, a positive GDD is added by SPM of the linearly chirped pulse, whether the initial chirp is up or down. On the other hand, SPM of a nonlinearly chirped pulse produces a TOD with the opposite sign to the initial TOD.

Figure 2. Third-Order Dispersion (TOD) of the output pulse $\Phi_{O U T}^{\prime \prime \prime}$ as a function of initial TOD $\Phi^{\prime \prime \prime}$ for various values of $B$ and initial GDD $\Phi^{\prime \prime}$ of (a) 10 , (b) -10 , and (c) 0 .

(a)

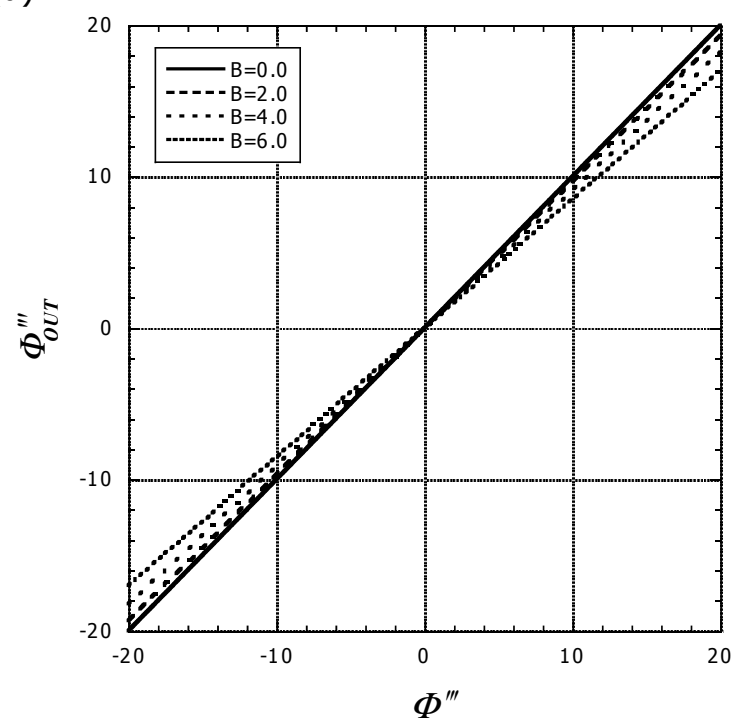

(b)

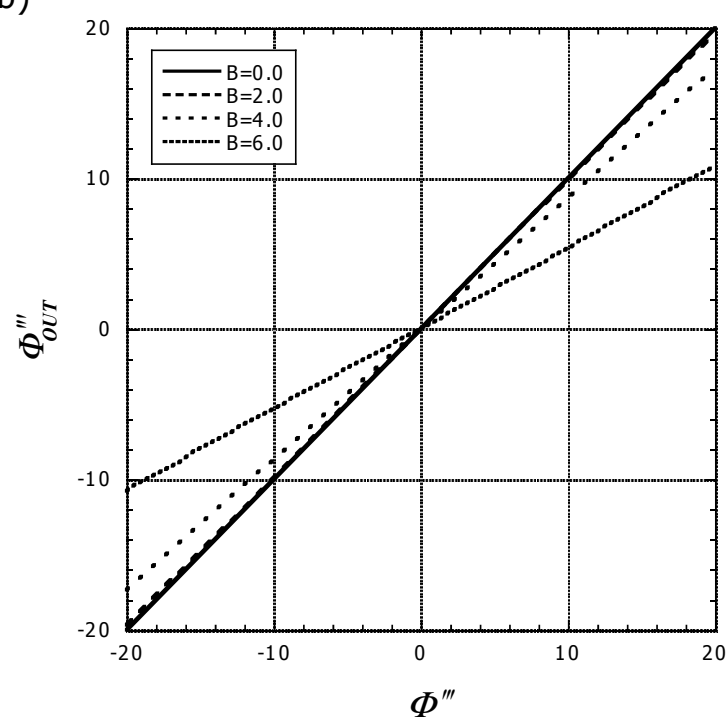

(c)

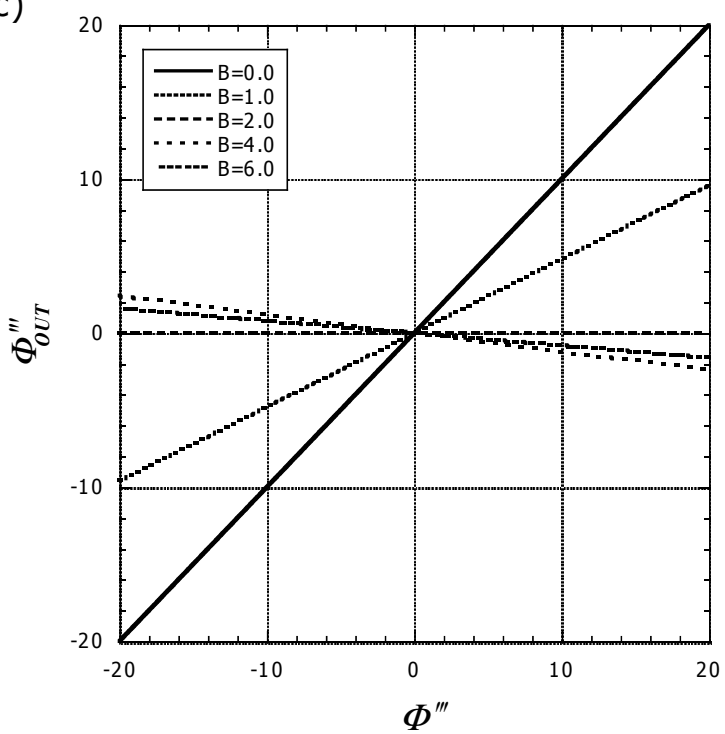

Summarizing the results shown in Figure 2, we can conclude that the TOD of the output pulse nearly equals the initial TOD when the $B$-integral is much smaller than the absolute initial GDD $\left|\Phi^{\prime \prime}\right|$. On the other hand, the TOD is near zero when the $B$-integral is much larger than the absolute initial GDD. 


\subsection{Effect of Self-Steepening}

In order to determine the effect of self-steepening, we compare the TOD of the output pulse as a function of initial TOD with and without self-steepening. Figure 3 is the same as Figure 2(c), except that it includes the effect of self-steepening. Although the difference can be seen in the zero-intercepts of each line, which shift slightly depending on the value of $B$, the feature is roughly the same as shown in Figure 2(c). It should be noted that the effect of self-steepening is negligible for large initial GDD, whether the pulse is up or down chirped.

Figure 3. Same as Figure 2(c), except the effect of self-steepening is included.

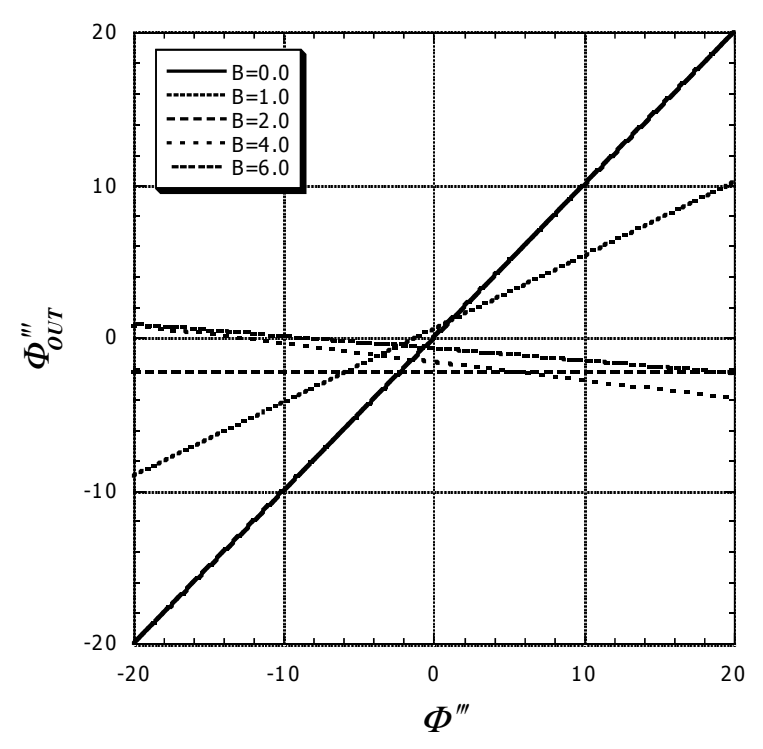

\section{Conclusions}

The use of chirped pulses in hollow-fiber pulse compression is proposed as a means to further increase the energy and intensity of few-cycle pulses. In this paper, we derived analytical expressions for chirped-pulse SPM, focusing on the relationship between the initial chirp and the dispersion remaining in the output pulse. The results show that a strongly chirped pulse with large initial GDD retains the initial TOD. On the other hand, the initial TOD decreases in a FTL or weakly chirped pulse. Such characteristics are convenient for dispersion compensation in the SPM-based pulse compression technique. It has generally been considered that pre-compensation of the chirp in an optical system including nonlinear processes such as SPM is difficult. However, the present result shows that dispersion can be roughly compensated for by a pair of prisms or chirped mirrors after a hollow fiber, while the fine-adjustment can be carried out with a programmable phase modulator prior to the hollow fiber, or even before a chirped-pulse amplifier.

\section{References}

1. Fork, R.I.; Cruz, C.H.B.; Becker, P.C.; Shank, C.V. Compression of optical pulses to six femtoseconds by using cubic phase compression. Opt. Lett. 1987, 12, 483-485. 
2. Nisoli, M.; De Silvestri, S.; Svelto, O. Generation of high energy 10 fs pulses by a new pulse compression technique. Appl. Phys. Lett. 1996, 68, 2793-2795.

3. Hentschel, M.; Kienberger, R.; Spielmann, Ch.; Reider, G.A.; Milosevic, N.; Brabec, T.; Corkum, P.; Heinzmann, U.; Drescher, M.; Krausz, F. Attosecond metrology. Nature 2001, 414, 509-513.

4. Suda, A.; Hatayama, M.; Nagasaka, K.; Midorikawa, K. Generation of sub-10-fs, 5-mJ optical pulses using a hollow fiber with a pressure gradient. Appl. Phys. Lett. 2005, 86, 111116:1-111116:3.

5. Laegsgaard, J.; Roberts, P.J. Dispersive pulse compression in hollow-core photonic bandgap fibers. Opt. Express 2008, 16, 9628-9644.

6. Park, J.; Lee, J.; Nam, C.H. Generation of 1.5 cycle 0.3 TW laser pulses using a hollow-fiber pulse compressor. Opt. Lett. 2009, 34, 2342-2344.

7. Bohman, S.; Suda, A.; Kanai, T.; Yamaguchi, S.; Midorikawa, K. Generation of 5.0 fs, $5.0 \mathrm{~mJ}$ pulses at $1 \mathrm{kHz}$ using hollow-fiber pulse compression. Opt. Lett. 2010, 35, 1887-1889.

(C) 2012 by the authors; licensee MDPI, Basel, Switzerland. This article is an open access article distributed under the terms and conditions of the Creative Commons Attribution license (http://creativecommons.org/licenses/by/3.0/). 